\title{
Video Article \\ Choice and No-Choice Assays for Testing the Resistance of A. thaliana to Chewing Insects
}

\author{
Martin De Vos ${ }^{1}$, Georg Jander ${ }^{1}$ \\ ${ }^{1}$ Boyce Thompson Institute for Plant Research, Cornell University \\ URL: https://www.jove.com/video/683 \\ DOI: doi:10.3791/683
}

Keywords: Plant Biology, Issue 15, Annual Review, Plant Resistance, Herbivory, Arabidopsis thaliana, Pieris rapae, Caterpillars, Butterflies, Jasmonic Acid, Glucosinolates

Date Published: 5/14/2008

Citation: De Vos, M., Jander, G. Choice and No-Choice Assays for Testing the Resistance of A. thaliana to Chewing Insects. J. Vis. Exp. (15), e683, doi:10.3791/683 (2008).

\section{Abstract}

Larvae of the small white cabbage butterfly are a pest in agricultural settings. This caterpillar species feeds from plants in the cabbage family, which include many crops such as cabbage, broccoli, Brussel sprouts etc. Rearing of the insects takes place on cabbage plants in the greenhouse. At least two cages are needed for the rearing of Pieris rapae. One for the larvae and the other to contain the adults, the butterflies. In order to investigate the role of plant hormones and toxic plant chemicals in resistance to this insect pest, we demonstrate two experiments. First, determination of the role of jasmonic acid (JA - a plant hormone often indicated in resistance to insects) in resistance to the chewing insect Pieris rapae. Caterpillar growth can be compared on wild-type and mutant plants impaired in production of JA. This experiment is considered "No Choice", because larvae are forced to subsist on a single plant which synthesizes or is deficient in JA. Second, we demonstrate an experiment that investigates the role of glucosinolates, which are used as oviposition (egg-laying) signals. Here, we use WT and mutant Arabidopsis impaired in glucosinolate production in a "Choice" experiment in which female butterflies are allowed to choose to lay their eggs on plants of either genotype. This video demonstrates the experimental setup for both assays as well as representative results.

\section{Video Link}

The video component of this article can be found at https://www.jove.com/video/683/

\section{Protocol}

\section{No Choice Experiment:}

1. Carefully enclose the Arabidopsis plant of interest in a plastic cup. In this case: Col-0 (WT) and a mutant impaired in the production of JA. Make sure you limit damage to the leaves. For that I use the back of this paintbrush.

2. Use a fine paintbrush to take a freshly hatched larvae from the colony on cabbage.

3. Carefully place the larva on the Arabidopsis plants.

4. Weight larva at day $4,7,10$. TIP: Grow your plants under short day conditions ( $8 \mathrm{hr}$ light) to increase vegetative growth (1 larva can fulfill its full life cycle on 1 plant).

\section{Choice Experiment:}

1. Two plants are selected, one of each genotype. Best is to grow plants in the same pot, to limit other factors, such as tray effects, etc.

2. Place the plants in a butterfly cage.

3. Select a fertile female. I usually catch a female that is actively laying eggs in the rearing colony.

4. Release the female in the cage with the choice of two plants for $24 \mathrm{hrs}$.

TIP 1: To eliminate an affect of learning, do not reuse females for subsequent choice tests.

TIP 2: One can only compare plants that have a similar growth phenotype. Alternatively, you could mount a leaf from each genotype on top of a Erlenmeyer flask and compare their attractiveness to a female.

Please visit Annual Reviews of Plant Biology for more information about this protocol and plant immunity to insect herbivory. 